\title{
Computational tools and resources for CRISPR/Cas 9 genome editing method
}

\begin{abstract}
CRISPR/Cas9, the popular genome editing method, has wide applications ranging from genetic engineering of plants, modifying bacterial genomes for production of therapeutic, agricultural, industrial bioproducts. It would be an understatement to claim that it has indeed revolutionized the field of gene and genome editing. To aid the researchers in designing the optimal sequences for CRISPR/Cas9 experiments, a multitude of bioinformatics tools and resources have been developed and are currently used. This review aims to provide an up to date list of tools, web servers, databases, and other resources that can be used for editing the genome. We hope that such a compilation will enable researchers to select the resources based on functionality, ease of use, availability, and specific research needs.
\end{abstract}

Keywords: CRISPR/Cas9, genome editing, bioinformatics, resources, database, computational tools
Volume 5 Issue 4 - 2017

\section{Ragothaman MYennmalli, Siddhant Kalra, Pulkit Anupam Srivastava, Vijay Kumar Garlapati \\ Department of Biotechnology and Bioinformatics, Jaypee} University of Information Technology, India

Correspondence: Vijay Kumar Garlapati, Department of Biotechnology and Bioinformatics, Jaypee University of Information Technology, Waknaghat, Distt: Solan, Himachal Pradesh- 173234, India, Tel 911792239225

Email shanepati@gmail.com

Received: February 15, 2017| Published: April 12, 2017
Abbreviations: CRISPR-Cas9, clustered regularly interspaced palindromic repeats-CRISPR associated proteins 9; crRNA, CRISPR RNA; DSB, DNA double helix break; sgRNA, single guide RNA; TALENs, transcription activator-like effectors nucleases; tracrRNA, transactivating CRISPR RNA; ZFNs, zinc finger nucleases

\section{Introduction}

From an evolutionary point of view, archaea and bacteria have evolved to survive and blossom as communities in active environments that are, in general, stressful and unpredictable. Specifically, the abundance of viruses attacking microorganisms is a constant threat; in addition, the mutation and recombination rates in viruses make them fast-evolving predators. To sustain against such predators, archaea and bacteria have evolved to have a new multilayered defense system that has been identified as CRISPR/Cas9. Consequently, many labs have tapped into the potential uses for this method and active research to unravel the mechanism has paved a unique path in the development of targeted genome editing techniques. ${ }^{2}$ Among the various genome editing methods, TALENs, ZFNs, and CRISPR-Cas9 are the three foremost methods. ${ }^{3}$

The advantage of CRISPR as a more reliable method is the ease in the creation of a break in the helical strand using RNA-guided nucleases that involve canonical base pairing between the target DNA region and the RNA designed for binding to it. These inducible alterations in the genome provide a potential avenue for not only transferring traits in livestock and crops, but also to rectify diseased genes for human therapeutics with application in gene therapy. Another advantage is the non-dependency on protein-based system such as ZFNs and TALENs. ${ }^{4}$

CRISPR-Cas9 system is made of three components, viz: a small non-coding RNA called tracrRNA, a 20 nucleotide containing RNA sequence called crRNA that binds to the target DNA, and a RNA sequence repeat of 19-22 bases; and a Cas protein encoding operon. For simplification of the system for targeted mutations, a sgRNA with unique restriction sites for specific insertion of the target by combining endogenous tracrRNA and crRNA. ${ }^{5}$
CRISPR-Cas9 gene editing technology can generate transformative medicines in health sector such as the production of antimicrobials, animal health and therapeutic bio products. In the case of agricultural biotechnology, the CRISPR-Cas9 technology aids in unraveling of both functional and phenotypical data about target plants and enhancing their resilience against drought and disease. Also, through breeding crops with higher yields. ${ }^{6}$ CRISPR-Cas9 technology is a valuable tool in biological research specifically to modify genes through knockout studies, inserting specific sequences, up-regulation and down-regulation of genes. The profound use of CRISPR-Cas9 technology has acknowledged by several researchers in generating accurate and efficient models of disease in cell lines, primary cells, and animal models. ${ }^{7}$ CRISPR-Cas9 technology provides a precise method to improve industrially important microbial strains as cell factories (Eg: fermentation) for production of food, pharmaceutical, and biofuel products. ${ }^{8}$

CRISPR's extensive applicability via gene editing and its easy rules for designing sgRNA has provided an excellent opportunity for bioinformatics researchers to get an insight of various aspects of CRISPR mechanism using computational approach and development of software that identifies potential sgRNA sequences in genomes. Although, several design tools, web servers, and databases exist not all of them are similar. Specifically, each has an advantage over the other. Hence, to make the job easier for researchers around the globe, there is a need to keep an eye for various CRISPR design software based on some of the potential advantages, such as functionality, ease to use, its availability and more specifically research needs.

\section{Software and web servers for designing sgRNA sequences}

Table 1 lists the currently available software (standalone and web server) that used for designing sgRNA for CRISPR/Cas9 experiments. While previous reports have listed the available software/web server for CRISPR/Cas9 experiments, some of the resources are inaccessible, for unknown reasons. For example, the web servers ZiFiT, Cas9Design, Jack Lin's CRISPR/Cas9 gRNA finder, and GT-Scan are no longer accessible for users. Among the 54 web servers listed in Table 
1, more than 20 resources are available for public use since 2015 , in comparison 13 webservers have been reported in 2016, and most recently one web server (CRISPR-RT) has been published in 2017.

Additionally, there are 5 software that can use in the standalone mode, by either running a Perl script (sgRNAcas9) or via using an

Table I List of software and web servers for designing sgRNA sequences.
R code (caRpools) (Table 2). There also desktop based applications (DESKGEN) that can be utilized by molecular biologists for designing sgRNA sequences. The potential drawback to the standalone versions could be software compatibility, ease of usage, and additionally, some may be available for a fee.

\begin{tabular}{|c|c|c|c|c|}
\hline S. no & Tool name & Standalone/ Web server & Link & Reference \\
\hline I & DESKGEN & Standalone & https://www.deskgen.com/landing/ & 9 \\
\hline 2 & sgRNAcas9 & Standalone/command-line & http://www.biootools.com/ & 10 \\
\hline 3 & caRpools & Standalone/ command-line & http://github.com/boutroslab/caRpools & 11 \\
\hline 4 & CRISPR Recognition Tool & Standalone & http://www.room220.com/crt/ & 12 \\
\hline 5 & CRISPRseek & Standalone & $\begin{array}{l}\text { http://bioconductor.org/packages/release/bioc/html/ } \\
\text { CRISPRseek.html }\end{array}$ & 13 \\
\hline 6 & CRISPRdirect & Web/online/graphic interface & http://crispr.dbcls.jp/ & 14 \\
\hline 7 & CRISPR RNA Configurator & Web/online/graphic interface & $\begin{array}{l}\text { http://dharmacon.gelifesciences.com/ ch/gene-editing/crispr- } \\
\text { rna-configurator/ }\end{array}$ & - \\
\hline 8 & CRISPRer & Web/online/graphic interface & http://jstacs.de/index.php/CRISPRer & - \\
\hline 9 & CRISPRTarget & Web/online/graphic interface & http://bioanalysis.otago.ac.nz/CRISPRTarget & 15 \\
\hline 10 & CRISPRfinder & Web/online/graphic interface & http://crispr.u-psud.fr/Server/ & 16 \\
\hline II & CRISPR gRNA Design tool & Web/online/graphic interface & https://www.dna20.com/eCommerce/cas9/input & - \\
\hline 12 & COD (Cas9 Online Designer) & Web/online/graphic interface & http://cas9.wicp.net/ & - \\
\hline 13 & CRISPR & Web/online/graphic interface & http://crispr.mit.edu/ & 17 \\
\hline 14 & Cas9 design & Web/online/graphic interface & http://cas9.cbi.pku.edu.cn/ & 18 \\
\hline 15 & sgRNA Designer & Web/online/graphic interface & $\begin{array}{l}\text { http://www.broadinstitute.org/rnai/public/ analysis-tools/ } \\
\text { sgrna-design }\end{array}$ & 19 \\
\hline 16 & CRISPR-GA & Web/online/graphic interface & http://crispr-ga.net & 20 \\
\hline 17 & Microhomology- Predictor & Web/online/graphic interface & http://www.rgenome.net/mich-calculator/ & 21 \\
\hline 18 & CRISPResso & Web/online/graphic interface & http://crispresso.rocks/ & 22 \\
\hline 19 & MAGeCK-VISPR & Web/online/graphic interface & https://bitbucket.org/liulab/mageck-vispr/ & 23 \\
\hline 20 & CrispRVariants & Web/online/graphic interface & https://github.com/markrobinsonuzh/CrispRVariants & 24 \\
\hline 21 & Cas-Designer & Web server & http://www.rgenome.net/cas-designer/ & 25,26 \\
\hline 22 & CRISPR-ERA & Web server & http://CRISPR-ERA.stanford.edu. & 27 \\
\hline 23 & CGAT & Web server & http://cbc.gdcb.iastate.edu/cgat/ & 28 \\
\hline 24 & E-CRISP & Web server & http://www.e-crisp.org/E-CRISP/ & 29 \\
\hline 25 & Cas-OFFinder & Web server & http://www.rgenome.net/cas-offinder/ & 30 \\
\hline 26 & CRISPRmap & Web server & http://rna.informatik.uni-freiburg.de/CRISPRmap/Input.jsp & 31 \\
\hline 27 & CHOPCHOP & Web server & https://chopchop.rc.fas.harvard.edu/ & 32 \\
\hline 28 & CRISPR design tool & Web server & https://www.atum.bio/eCommerce/cas9/input & 33 \\
\hline 29 & CroplT & Web server & $\begin{array}{l}\text { http://cheetah.bioch.virginia.edu/AdliLab/CROP-IT/ } \\
\text { homepage.html }\end{array}$ & 34 \\
\hline 30 & CasFinder & Web server & http://arep.med.harvard.edu/CasFinder/ & 35 \\
\hline 31 & ZiFiT & Web server & http://zifit.partners.org/ZiFiT/ & 36 \\
\hline 32 & CasOT & Web server & http://eendb.zfgenetics.org/casot/ & 37 \\
\hline 33 & Cas9-Design & Web server & http://cas9.cbi.pku.edu.cn/ & 38 \\
\hline 34 & CCTop & Web server & http://crispr.cos.uni-heidelberg.de/ & 39 \\
\hline
\end{tabular}


Table Continued....

\begin{tabular}{|c|c|c|c|c|}
\hline S. no & Tool name & Standalone/Web server & Link & Reference \\
\hline 35 & COSMID & Web server & https://crispr.bme.gatech.edu/ & 40 \\
\hline 36 & sgRNA Scorer & Web server & https://crispr.med.harvard.edu/sgRNAScorer/ & 41 \\
\hline 37 & EuPaGDT & Web server & http://grna.ctegd.uga.edu/ & 42 \\
\hline 38 & CRISPR-RT & Web server & http://bioinfolab.miamioh.edu/CRISPR-RT/ & 43 \\
\hline 39 & BreakingCas & Web server & http://bioinfogp.cnb.csic.es/tools/breakingcas/index.php & 44 \\
\hline 40 & CRISPR-DO & Web server & http://cistrome.org/crispr/ & 45 \\
\hline 41 & $\begin{array}{l}\text { CRISPR/Cas9 target online } \\
\text { predictor }\end{array}$ & Web server & http://crispr.cos.uni-heidelberg.de/ & 46 \\
\hline 42 & ProtospacerWB & Web server & http://www.protospacer.com/ & 47 \\
\hline 43 & CRISPR-P & Web server & http://cbi.hzau.edu.cn/crispr/ & 48 \\
\hline 44 & CRISPy & Web server & http://staff.biosustain.dtu.dk/laeb/crispy/ & 49 \\
\hline 45 & CLD & Web server & https://github.com/boutroslab/cld & 50 \\
\hline 46 & CRISPOR & Web server & http://crispor.tefor.net/ & 51 \\
\hline 47 & Off-Spotter & Web server & https://cm.jefferson.edu/Off-Spotter/ & 52 \\
\hline 48 & SSC & Web server & http://crispr.dfci.harvard.edu/SSC/ & 53 \\
\hline 49 & CT-Finder & webserver & http://bioinfolab.miamioh.edu/ct-finder/ & 54 \\
\hline 50 & CRISPETa & Web server & http://crispeta.crg.eu/ & 55 \\
\hline 51 & WU-CRISPR & Web server & http://crispr.wustl.edu/ & 56 \\
\hline 52 & CRISPRscan & Web server & http://www.crisprscan.org/ & 57 \\
\hline 53 & CRISPR-ERA & Web server & http://crispr-era.stanford.edu/ & 58 \\
\hline 54 & Azimuth & Web server & https://www.microsoft.com/en-us/research/project/azimuth/ & 59 \\
\hline 55 & SSFinder & Web server & https://github.com/alaindomissy/ssfinder & 60 \\
\hline 56 & CRISPR multitargeter & Web server & http://www.multicrispr.net/ & 61 \\
\hline 57 & flyCRISPR & Web server & http://tools.flycrispr.molbio.wisc.edu/targetFinder/ & 62 \\
\hline 58 & sgRNAcas9 & Web server & http://www.biootools.com/col.jsp?id=103 & 63 \\
\hline 59 & Benchling & Web server & https://benchling.com/ & -- \\
\hline 60 & AGEseq & Web server & http://aspendb.uga.edu:8085/ & 64 \\
\hline 61 & MAGeCK & Web server & https://sourceforge.net/p/mageck/wiki/Home/ & 65 \\
\hline 62 & $\begin{array}{l}\text { Jack Lin's CRISPR/Cas9 gRNA } \\
\text { finder }\end{array}$ & Web server & http://spot.colorado.edu/_slin/cas9.html & 66 \\
\hline 63 & GT-Scan & Web server & http://gt-scan.braembl.org.au/gt-scan/ & 67 \\
\hline 64 & PhytoCRISP-Ex & Web server, standalone & http://www.phytocrispex.biologie.ens.fr/CRISP-Ex/ & 68 \\
\hline 65 & CRISPR design & Web server & http://crispr.mit.edu/ & - \\
\hline
\end{tabular}

Table 2 List of databases for designing sgRNA sequences

\begin{tabular}{llll}
\hline $\begin{array}{l}\text { S. } \\
\text { no }\end{array}$ & $\begin{array}{l}\text { Tool } \\
\text { name }\end{array}$ & Link & Reference \\
\hline I & WGE & http://www.sanger.ac.uk/htgt/wge/ & 69 \\
2 & Cas- & http://www.rgenome.net/cas-database/ & 70 \\
& Database & http://crdd.osdd.net/servers/crisprge/. & 71 \\
3 & CrisprGE & ht/ & 72 \\
4 & CRISPRdb & http://crispr.u-psud.fr/crispr & 73 \\
5 & COSMID & https://crispr.bme.gatech.edu/ & 7 \\
\hline
\end{tabular}

\section{Databases for designing CRISPR/Cas9 genome editing}

Almost of the tools listed in Table 1 most likely have an inbuilt database for designing sgRNA sequences. However, for the sake of simplicity, we have listed 5 resources as "databases", which are have collated the sgRNA sequences and they can be easily accessed. We have included COSMID in both web server and database as it is an advanced resource available for users. ${ }^{74}$

\section{Discussion}

Microbes are the cell factories for the production of therapeutic and industrial biological products. Until recently, metabolic engineering 
is at the forefront as a paradigm changing research area by which genomes can edit as desired. Nowadays, CRISPR and its associated proteins (Cas) are currently popular for precision genome editing in many organisms. Based on the global market statistics, the genome editing market is expected to touch around USD 5.54 Billion by the end of 2021 with a CAGR of $14.3 \%$. Among different genome editing technologies the CRISPR segment stands alone with the largest share of the global genome editing market due to the ease usage of the technology. ${ }^{75}$

\section{Conclusion}

In this mini-review, we have highlighted an updated and currently accessible compilation of software (standalone and web server) and databases that are used for CRISPR/Cas9 genome editing method. With the availability of multiple resources for sgRNA design, the prediction accuracy and validated benchmark sets are utmost important for efficient targeted genome editing research.

\section{Acknowledgements}

The authors acknowledge the resource facilities provided by the Jaypee University of Information Technology, Waknaghat, HP173234, India for executing the present mini-review.

\section{Conflict of interest}

The author declares no conflict of interest.

\section{References}

1. Choi KR, Lee SY. CRISPR technologies for bacterial systems: current achievements and future directions. Biotechnol Adv. 2016;34 (7):11801209.

2. Blin K, Pedersen LE, Weber T, et al. CRISPy-web: an online resource to design sgRNAs for CRISPR applications. Synth Syst Biotechnol. 2016;1:118-121.

3. Kanchiswamy CN, Maffei M, Malnoy M, et al. Fine-tuning next-generation genome editing tools. Trends Biotechnol. 2016;34(7):562-574.

4. Singh V, Braddick D, Dhar PK. Exploring the potential of genome editing CRISPR-Cas9 technology. Gene. 2017;599:1-18.

5. Quétier F. The CRISPR-Cas9 technology: Closer to the ultimate toolkit for targeted genome editing. Plant Sci. 2016;242:65-76.

6. Liu D, Hu R, Palla KJ, et al. Advances and perspectives on the use of CRISPR/Cas9 systems in plant genomics research. Curr Opin Plant Biol. 2016;30:70-77.

7. Luo J. CRISPR/Cas9: from genome engineering to cancer drug discovery. Trends in cancer. 2016;2(6):313-324.

8. Jakočiūnas T, Jensen MK, Keasling JD. CRISPR/Cas9 advances engineering of microbial cell factories. Metab Eng. 2016;34:44-59.

9. Li Y, Park AI, Mou H, et al. A versatile reporter system for CRISPR-mediated chromosomal rearrangements. Genome Biol. 2015;16:111.

10. Xie S, Shen B, Zhang C, et al. sgRNAcas9:a software package for designing CRISPR sgRNA and evaluating potential off-target cleavage sites. PLoS One. 2014;9:e100448.

11. Winter J, Breinig M, Heigwer F, et al. caRpools: an R package for exploratory data analysis and documentation of pooled CRISPR/Cas9 screens. Bioinformatics. 2016;32(4):632-634

12. Bland C, Ramsey TL, Sabree F, et al. CRISPR recognition tool (CRT) a tool for automatic detection of clustered regularly interspaced palindromic repeats. BMC Bioinformatics. 2007;8(1):209.
13. Zhu LJ, Holmes BR, Aronin N, et al. CRISPRseek: a Bioconductor package to identify target-specific guide RNAs for CRISPR-Cas9 genomeediting systems. PLoS one. 2014;9:e108424.

14. Naito Y, Hino K, Bono H, Ui-Tei K. CRISPRdirect: software for designing CRISPR/Cas guide RNA with reduced off-target sites. Bioinformatics. 2014;31(7):1120-1123.

15. Biswas A, Gagnon JN, Brouns SJ, et al. CRISPRTarget: bioinformatic prediction and analysis of crRNA targets. RNA Biol. 2013;10(5):817827.

16. Grissa I, Vergnaud G, Pourcel C. CRISPR Finder: a web tool to identify clustered regularly interspaced short palindromic repeats. Nucleic Acids Res. 2007;35:W52-W57.

17. Hsu PD, Scott DA, Weinstein JA, et al. DNA targeting specificity of RNA-guided Cas9 nucleases. Nat Biotechnol. 2013;31(9):827-832.

18. Ma M, Ye AY, Zheng W, et al. A guide RNA sequence design platform for the CRISPR/Cas9 system for model organism genomes. Biomed Res Int. 2013;2013:270805.

19. Doench JG, Fusi N, Sullender M, et al. Optimized sgRNA design to maximize activity and minimize off-target effects of CRISPR-Cas9. Nat Biotechnol. 2016;34(2):184-191.

20. Güell M, Yang L, Church GM. Genome editing assessment using CRISPR Genome Analyzer (CRISPR-GA). Bioinformatics. 2014;30(20):29682970.

21. Bae S, Kweon J, Kim HS, et al. Microhomology-based choice of Cas9 nuclease target sites. Nat Methods. 2014;11(5):705-706.

22. Pinello L, Canver MC, Hoban MD, et al. Analyzing CRISPR genomeediting experiments with CRISPResso. Nat Biotechnol. 2016;34(7):695697.

23. Li W, Köster J, Xu H4, et al. Quality control, modeling, and visualization of CRISPR screens with MAGeCK-VISPR. Genome Biol. 2015;16:113.

24. Lindsay H, Burger A, Biyong B, et al. CrispRVariants charts the mutation spectrum of genome engineering experiments. Nat Biotechnol. 2016;34(7):701-702.

25. Park J, Bae S, Kim JS. Cas-Designer: a web-based tool for choice of CRISPR-Cas9 target sites. Bioinformatics. 2015;31(24):4014-4016.

26. Bae S, Kweon J, Kim HS, et al. Microhomology-based choice of Cas9 nuclease target sites. Nat Methods. 2014;11(7):705-706.

27. Liu H, Wei Z, Dominguez A, et al. CRISPR-ERA: a comprehensive design tool for CRISPR-mediated gene editing, repression and activation. Bioinformatics. 2015;31(22):3676-3678.

28. Brazelton VA Jr, Zarecor S, Wright DA, et al. A quick guide to CRISPR sgRNA design tools. GM Crops Food. 2015;6(4):266-276.

29. Doench JG, Fusi N, Sullender M, et al. Optimized sgRNA design to maximize activity and minimize off-target effects of CRISPR-Cas9. Nat Biotechnol. 2016;34(2):184-191.

30. Bae S, Park J, Kim JS. Cas-OFFinder: a fast and versatile algorithm that searches for potential off-target sites of Cas9 RNA-guided endonucleases. Bioinformatics. 2014;30(10):1473-1475.

31. Mangericao TC, Peng Z, Zhang X. Computational prediction of CRISPR cassettes in gut metagenome samples from Chinese type-2 diabetic patients and healthy controls. BMC Syst Biol. 2016;10(Suppl 1):5.

32. Labun K, Montague TG, Gagnon JA, et al. CHOPCHOP v2: a web tool for the next generation of CRISPR genome engineering. Nucleic Acids Res. 2016;44(Web Server issue):W272-W276.

33. Hsu PD, Scott DA, Weinstein JA, et al. DNA targeting specificity of RNA-guided Cas9 nucleases. Nat Biotechnol. 2013;31(9):827-832. 
34. Singh R, Kuscu C, Quinlan A, et al. Cas9-chromatin binding information enables more accurate CRISPR off-target prediction. Nucleic Acids Res. 2015;43(18):e118.

35. Aach J, Mali P, Church GM. CasFinder: flexible algorithm for identifying specific Cas9 targets in genomes. BioRxiv. 2014;005074.

36. Sander JD, Maeder ML, Reyon D, Vet al. ZiFiT (Zinc Finger Targeter): an updated zinc finger engineering tool. Nucleic Acids Res. 2010;38:W462W468.

37. Xiao A, Cheng Z, Kong L, et al. CasOT: a genome-wide Cas9/gRNA off-target searching tool. Bioinformatics. 2014;30(8):1180-1182.

38. Brazelton VA Jr, Zarecor S, Wright DA, et al. A quick guide to CRISPR sgRNA design tools. GM Crops Food. 2015;6(4):266-276.

39. Stemmer M, Thumberger T, del Sol Keyer M, et al. CCTop: an intuitive, flexible and reliable CRISPR/Cas9 target prediction tool. PLOS One. 2015;10(4):e0124633.

40. Cradick TJ, Qiu P, Lee CM, et al. COSMID:A Web-based tool for identifying and validating CRISPR/Cas off-target sites. Mol Ther Nucleic Acids. 2014;3(12):e214.

41. Chari R, Mali P, Moosburner M, et al. Unraveling CRISPR-Cas9 genome engineering parameters via a library-on-library approach. Nat Methods. 2015;12(9):823-826.

42. Peng D, Tarleton R. EuPaGDT: a web tool tailored to design CRISPR guide RNAs for eukaryotic pathogens. Microbial Genomics. 2015;1(4):1

43. Zhu H, Richmond E, Liang C. CRISPR-RT: A web service for designing CRISPR-C2c2 crRNA with improved target specificity. BioRxiv. $2017 ; 099895$.

44. Oliveros JC, Franch M, Tabas-Madrid D, et al. Breaking-Cas-interactive design of guide RNAs for CRISPR-Cas experiments for ENSEMBL genomes. Nucleic Acids Res. 2016;44(W1):W267-W271.

45. Ma J, Köster J, Qin Q, et al. CRISPR-DO for genome-wide CRISPR design and optimization. Bioinformatics. 2016;32(21):3336-3338.

46. Stemmer M, Thumberger T, Del Sol Keyer M, et al. CCTop:An Intuitive, Flexible and Reliable CRISPR/Cas9 Target Prediction Tool. PLoS One. 2015;10(4):e0124633.

47. MacPherson CR, Scherf A. Flexible guide-RNA design for CRISPR applications using Protospacer Workbench. Nat Biotechnol. 2015;33(8):805-806.

48. Lei Y, Lu L, Liu HY, et al. CRISPR-P:a web tool for synthetic single-guide RNA design of CRISPR-system in plants. Mol Plant. 2014;7(9):1494-1496.

49. Ronda C, Pedersen LE, Hansen HG, et al. Accelerating genome editing in CHO cells using CRISPR Cas9 and CRISPy, a web-based target finding tool. Biotechnol Bioeng. 2014;111(8):1604-1616.

50. Heigwer F, Zhan T, Breinig M, et al. CRISPR library designer (CLD):software for multispecies design of single guide RNA libraries. Genome Biol. 2016;17:55.

51. Haeussler M, Schönig K2, Eckert H3, et al. Evaluation of off-target and on-target scoring algorithms and integration into the guide RNA selection tool CRISPOR. Genome Biol. 2016;17(1):148.

52. Pliatsika V, Rigoutsos I. "Off-Spotter": very fast and exhaustive enumeration of genomic lookalikes for designing CRISPR/Cas guide RNAs. Biol Direct. 2015;10:4.

53. Xu H, Xiao T, Chen $\mathrm{CH}$, et al. Sequence determinants of improved CRISPR sgRNA design. Genome Res. 2015;25(8):1147-1157.

54. Zhu H, Misel L, Graham M, et al. CT-Finder: A web service for CRISPR optimal target prediction and visualization. Sci Rep. 2016;6:25516.
55. Pulido-Quetglas C, Aparicio-Prat E, Arnan C, et al. Scalable design of paired CRISPR GUIDE RNAs for genomic deletion. bioRxiv. $2016 ; 052795$.

56. Wong N, Liu W, Wang X. WU-CRISPR: characteristics of functional guide RNAs for the CRISPR/Cas9 system. Genome Biol. 2015;16:218.

57. Moreno-Mateos MA, Vejnar CE, Beaudoin JD, et al. CRISPRscan: designing highly efficient sgRNAs for CRISPR-Cas9 targeting in vivo. Nat Methods. 2015;12(10):982-988.

58. Liu H, Wei Z, Dominguez A, et al. CRISPR-ERA: a comprehensive design tool for CRISPR-mediated gene editing, repression and activation. Bioinformatics. 2015;31(22):3676-3678.

59. Fusi N, Smith I, Doench J, et al. In Silico Predictive Modeling of CRISPR/Cas9 guide efficiency. BioRxiv. 2015;021568.

60. Upadhyay SK, Sharma S. SS Finder: high throughput CRISPR-Cas target sites prediction tool. BioMed Res Int. 2014;2014:742482.

61. Prykhozhij SV, Rajan V, Gaston D, et al. CRISPR multitargeter: a web tool to find common and unique CRISPR single guide RNA targets in a set of similar sequences. PloS One. 2015;10(3):e0119372.

62. Gratz SJ, Ukken FP, Rubinstein CD, et al. Highly specific and efficient CRISPR/Cas9-catalyzed homology-directed repair in Drosophila. Genetics. 2014;196(4):961-971.

63. Xie S, Shen B, Zhang C, et al. sgRNAcas9: a software package for designing CRISPR sgRNA and evaluating potential off-target cleavage sites. PloS one. 2014;9(6):e100448.

64. Xue LJ, Tsai CJ. AGEseq: analysis of genome editing by sequencing. Mol Plant. 2014;8(9):1428-1430.

65. Li W, Xu H, Xiao T, et al. MAGeCK enables robust identification of essential genes from genome-scale CRISPR/Cas9knockout screens. Genome Biol. 2014;15(12):554.

66. Prashant Mali, Luhan Yang, Kevin M Esvelt, et al. RNA-guided human genome engineering via Cas9. Science. 2013;339(6121):823-826.

67. O’Brien A, Bailey TL. GT-Scan: identifying unique genomic targets. Bioinformatics. 2014;30(18):2673-2675.

68. Achal Rastogi A, Omer Murik, Chris Bowler, et al. PhytoCRISP-Ex: a web-based and stand-alone application to find specific target sequences for CRISPR/CAS editing. BMC Bioinformatics. 2016;17(1):261.

69. Hodgkins A. WGE: aCRISPRdatabaseforgenome engineering. Bioinformatics. 2015;31:3078-3080.

70. Park J, Kim JS, Bae S. Cas-Database: web-based genome-wide guide RNA library design for gene knockout screens using CRISPR-Cas9. Bioinformatics. 2016;32(13):2017-2023.

71. Kaur K, Tandon H, Gupta AK, et al. CrisprGE: a central hub of CRISPR/ Cas-based genome editing. Database. 2015;2015:bav055.

72. Grissa I, Vergnaud G, Pourcel C. The CRISPRdb database and tools to display CRISPRs and to generate dictionaries of spacers and repeats. BMC Bioinform. 2007;8:172.

73. Cradick TJ, Qiu P, Lee CM, et al. COSMID: a web-based tool for identifying and validating CRISPR/Cas off-target sites. Mol Ther Nucleic Acids. 2014;3(12):e214.

74. Chuai GH, Wang QL, Liu Q. In-Silico meets In Vivo: towards computational CRISPR-Based sgRNA design. Trend Biotechnol. 2014;35(1):1221.

75. http://www.marketsandmarkets.com/PressReleases/genome-editing-engineering.asp 